\title{
Practicality Verification of an Application Virtualization System Assuming Use of BYOD Environment
}

\author{
Toyohiro Hayashi, Yutaka Fukuda, Akihiro Satoh, Kyoko Kai, Noriyuki Kushiro \\ Information Science and Technology Center, Kyushu Institute of Technology, Fukuoka, Japan \\ Email: toyohiro@isc.kyutech.ac.jp
}

How to cite this paper: Hayashi, T., Fukuda, Y., Satoh, A., Kai, K., \& Kushiro, N. (2020). Practicality Verification of an Application Virtualization System Assuming Use of BYOD Environment. Open Journal of Social Sciences, 8, 482-492. https://doi.org/10.4236/jss.2020.83040

Received: March 10, 2020 Accepted: March 28, 2020 Published: March 31, 2020

\begin{abstract}
From 2019, We - Kyushu Institute of Technology started to bring and use students' laptops (called "BYOD on education") in some lectures. In the same timing, we have replaced our information systems for education and research use (we call "Education systems"). The new system is turned to support our BYOD environment on education scene. For one thing that, "Application virtualization system" has been adopted. It behaves as "the application was installed on PCs" without some installation procedures. It can be expected to enhance lectures such as some exercise using software. Before providing application virtualization system, some practicality verification is needed. We adopt this system to 30 different software for education and research use. After verification, we judged that 25 of 30 software can be adopting for BYOD environment on education scene.
\end{abstract}

\section{Keywords}

BYOD on Lectures, Application Virtualization System, Application Delivery, Cloudpaging

\section{Bring and Use Students' Laptops on Our Institute}

BYOD (Bring Your Own Device) is a trend way to bring own information equipment and use for business scene. Recently, BYOD is often adopting for education on universities, there are some reports of BYOD by using students' laptops on national university of Japan (Ohashi et al., 2018). On the other hand until 2017, in our institute, Kyushu Institute of Technology, information related lectures are opened on "PC classrooms" that PC terminal (Windows and Ubuntu can use) are equipped. In the latest system (2014-2018), Ubuntu Desktop was 
operated mainly on a PC terminal, and collective education such as exercises was opened. This is the reason that some performances (CPU speed, RAM capacity etc.) are under spec for information related lectures on a college of technology, so we avoid adopt of BYOD for a long time.

However, the trend of adopting became stronger around 2016.Some middle class of laptops released on 2018, benchmarks of computation (CPU speed etc.) are same or upper than the PC terminal (desktop style PC) on our classrooms introduced on 2014. In addition, these laptops have rich capacity of batteries, so it can be worked all of day on fully charged (Tomishige et al., 2019).

Actually, over than $90 \%$ of students in our institute have own laptops. Focusing on information infrastructure and services for our institute's members. Over 400 of Wi-Fi access points in our campus are set, so users can access to campus network from almost buildings/classrooms. Essential tools for taking lecture (such as LMS, course registration system) are built as web application, and we utilize many public IaaS such as e-mail system, online storage system. Student can use many tools via Wi-Fi and own laptops.

As described above, performances of laptops, ownership ratio of students' laptops, status of information infrastructure are higher degree.

So BYOD by using students' laptops were considered by our executive committee in 2016. Finally, we decided that starts BYOD from 2018 (Kyushu Institute of Technology, 2019) (Kai et al., 2019).

\section{Preparation of Information Infrastructure, Web Services, Software Licenses for Education}

In order to educate utilized BYOD smoothly, we think the following should be prepared. These are:

1) Information network infrastructure such as Wi-Fi APs, high speed campus network,

2) Web services for education,

3) Comprehensive software licensing and management.

Status of these preparations in our institute is below.

1) Information network infrastructure

High speed information network is prepared as campus network. Connection speed about inter-building in campus is $10 \mathrm{Gbps}-40 \mathrm{Gbps}$ range. In all of classroom, Wireless LAN (Wi-Fi, IEEE802.11ax or IEEE802.11ac) access points are set.

There are about 470 APs at Sep.2019. A network connection via Wi-Fi is provided as "Information outlet service", and this access is controlled by IEEE802.1X (EAP).

All of staff and student can connect campus network by theirs unified account (Fukuda et al., 2019).

2) Web services for education

Most of services for education and research use are deployed as Web Application, their services can be accessed by generic WWW browsers with some exten- 
sions. We provide web services for students such as LMS (Moodle, OSS), course registration system (Live Campus, NTT Data Inc.), self assessment of learning recording system (Custom made), e-mail and online storage (Office 365 A1, Microsoft), online manual of information infrastructure and services (published by Joomla (CMS)).

3) Comprehensive software licensing and management

We provide comprehensive licensing and management for software that will be in high demand in lectures and exercises. All of staff and student can install and use their software in own PCs under controlled condition. For example, major Microsoft products (Windows OS, Office suite, Visual Studio, etc.), endpoint Anti-Virus products, Engineering software (MATLAB, Mathematica) are provided as comprehensive.

\section{Supporting BYOD on Education by "Education Systems"}

To support information related lectures, education, and researches in our institute, we have exclusive information systems named "education systems". These are replaced every 4 - 5 years, and these are aimed an advanced architecture in every replaced scene. But basically, these mainly provided an environment using PC terminals (host terminal, UNIX terminal, net boot PC) in classrooms.

Recent replacing timing was set to 2017-2019. Concretely, it was planned to start design in 2017, start building systems in 2018, and switch to new system and start operation in April 2019.

This replacing timing will overlap with adopt BYOD (starts from 2018) on our institute. So commission and executive committee has suggested that education systems should support BYOD. Based on this suggestion, education systems are turned to support our BYOD environment on education scene. Concretely, deploying number of PC terminals in classrooms will be reduced to $50 \%$. On the other hand, providing high performance and availability web services, application platforms,

VM infrastructure, storage system is pushing ahead (Figure 1) (Hayashi et al., 2019).

\section{The Modern Application Virtualization System}

In some lectures, exercise format will be applied by using particular engineering application. In our institute, programming language (compiler), CAD, scientific computing platform are often used. To prepare an environment for providing these applications, below approaches has been adopted in the past.

1) Installing particular application to OS images for PC terminal

2) Preparation VDI (Virtual Desktop Infrastructure) environment for execution of particular application

3) Distribution of essentials for installs particular application (installer, license files etc.) to students

Exercise in every lecture can be done which approach is adopted. On the other hand, there are some problems on service operation. 


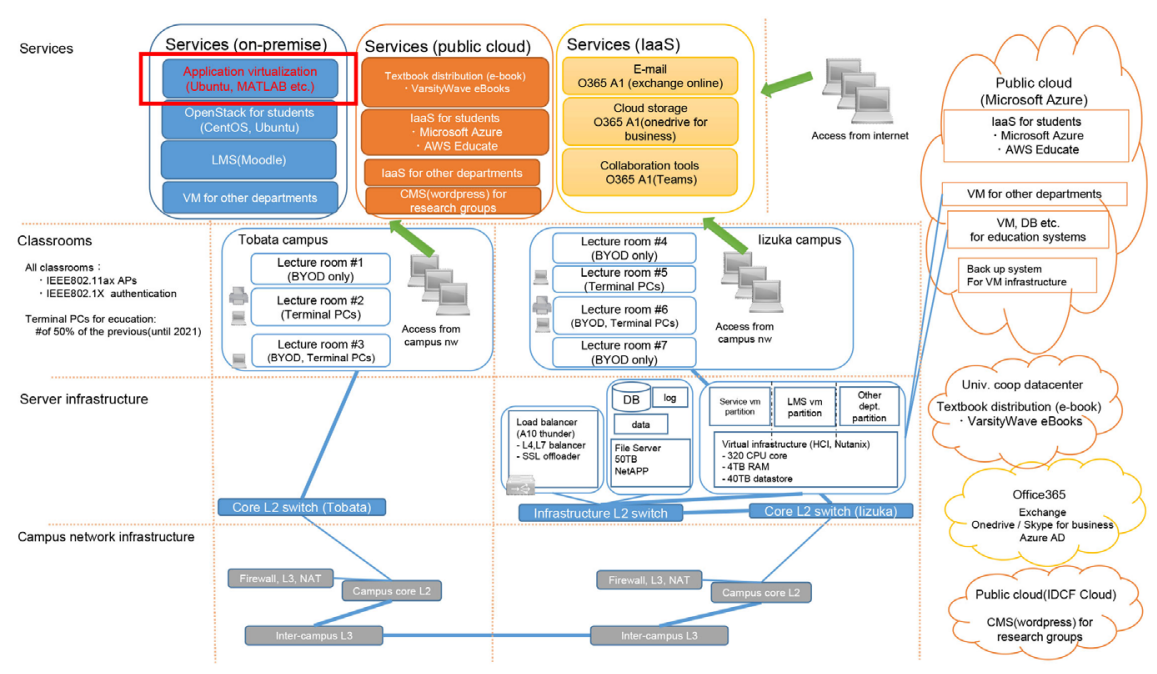

Figure 1. System configuration diagram of education systems (since 2019-).

In approach 1, making up and operation check test of OS images are required. In addition, this approach is applied only PC terminal, it is too limitedly.

In approach 2, particular application can be used from various remote terminals than approach 1. But in generally, operation of VDI service will be taken high operation cost, some application cannot be executed on VDI in license issue. And the weakest point is that performances of PC terminals or students' laptops do not contribute for running applications on VDI.

In approach 3, by taking software installer or license files to students will cause illegal taken out to outside, access control will be difficult, and inventory of software (uninstalling of software after graduation is needed, etc.) will be difficult. These will be risk of license management.

To resolve above problems, we have applied "Application virtualization system" in newer education systems. In next Section 3.1, we will describe more detail.

\subsection{Essentials of Application Virtualization System}

Application virtualization system will package (called "virtualization" or "encapsulation") essentials (such as Files, Device drivers, environment variables, registry information) to execute an application, and deliver packages to users' computing environment such as PC terminal, users' own PCs.

In users' computing environment, agent software for Application virtualization system behaves as if the delivered essentials were installed. Thus, an application can execute normally without installation process on users' environment. It can be expected to avoid installation hassle.

Typical software/hardware configuration of application virtualization system (shown on Figure 2) will be below elements.

1) Packaging software/dedicated PC

Packaging software detects execution of software installer, file allocation, registry creation etc. These detected elements are captured and packaged as 


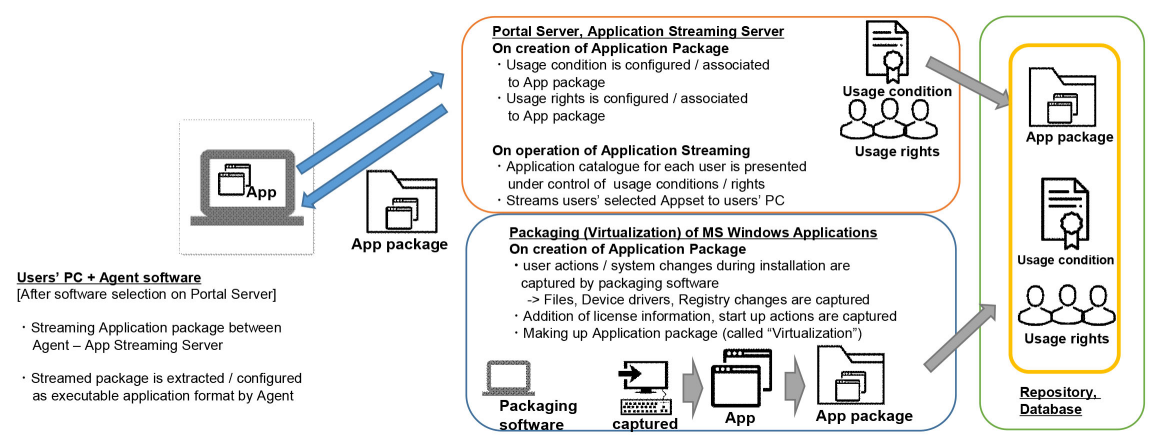

Figure 2. Typical configuration of application virtualization system.

"Application package". Packaging software and installer of application which users want to install should run on dedicated PC.

2) Portal server, Application streaming server

It provides functions for software (application package) selection web portal, delivery an chosen application package to users' environment. In app streaming server, usage condition (expiration date of application etc.) and usage rights (accessible users, computers etc.) are set for each application package. In addition, some products have functions about control delivery method for each package (delivery as "demand streaming" or "download" etc.).

3) Agent Software

Agent software will install on users' environment. It cooperates with application streaming server to control application execution. And it extracts application package and manipulate file system/registry of users' environment to behave as "an application is installed on users' environment directly".

By adopting an application virtualization system, an application can be executed on users' PCs natively. In addition, access control and license management for applications will be guaranteed. Making up of OS images, preparation of VDI environment, distribution of installer for applications are not needed. Problems described in the previous section are expected to be solved. After all, the approach of "an application can be executed on users' PCs natively" has a higher affinity for BYOD on education. It is an effective service to support BYOD.

But in fact, application virtualization system is not newer solution. It already existed in the early 2000s, and was introduced in our educational system operated at 2009-2013.It was used to distribute CAD software to classroom PC terminals (Nakamura, 2010). However, the system has serious restrictions, such as installation drive cannot be selected (cannot be installed on the $\mathrm{C}$ drive), and some of device drivers could not be included to application package.

\subsection{Our Selection: Numecent Cloudpaging: A Modern System}

As an implementation of application virtualization system to support BYOD, we applied Cloudpaging (by Numecent Inc.) for our educational system operated at 2019 (Numecent Inc., 2018). An system configuration is shown on Figure 3. 




Figure 3. Configuration on application virtualization system on education systems.

System configuration is almost same as other application virtualization systems. In this system, it is noted that the development cost is applied to the application packaging method. It contributes to the improvement of the packaging success rate.

The application package on Cloudpaging is called "Appset". In AppSet, capturing of low level device drivers into application package, control of allocation files (Whether to prioritize existing or delivered files/execute files, called "Layer"), granting privilege escalation to executable files in AppSet (it requires changing Windows Defender settings) are supported. These features contribute for rating up of packaging success rate.

On streaming server, setting of usage condition (called "Meta license") such as usable number of AppSet, delivery method (download or streaming) are supported. In addition, settings of usage right management (for users/groups/PCs, called "Principal") for using permission of Appset are also supported. These will be contribute of access control and license management for application. Major parameters on meta license and Principal is shown on Figure 4.

\section{Utilization of Cloudpaging in BYOD on Education}

By using Cloudpaging system, expected that distribution of software and materials used in classes, and provision of an self study environment will be made easier for students' own laptops. It will contribute to enhance lectures such as some exercise using software. But the first, we define some policies for distribution of software.

These are: 1) What applications should be prepare, 2) Parameters for Appset (These are related to license control etc.).

Regarding policies for distribution of software, the usage form BYOD environment in our institute is considered.

As follows,

1) Comprehensive license contract software can be installed on the student's laptops, but strict license management is required, 


\begin{tabular}{|c|c|c|c|}
\hline $\begin{array}{l}\text { Meta license: } \\
\text { usage condition }\end{array}$ & \multicolumn{2}{|l|}{ Behavior } & Example scenario \\
\hline Maximum Seats & \multicolumn{2}{|l|}{$\begin{array}{l}\text { Set a avalable number of AppSet (called "Sear"). } \\
\text { If t texceeds, AppSet will not be delivered }\end{array}$} & $\begin{array}{l}\text { Control the number of applications that can be started according to the } \\
\text { software contract }\end{array}$ \\
\hline Exipration Time & \multicolumn{2}{|l|}{$\begin{array}{l}\text { Set an expiration date of AppSet } \\
\text { If it exceeds, AppSet will not be delivered }\end{array}$} & Inhibit execution of sofware after student has been graduated \\
\hline Offlline Duration & \multicolumn{2}{|c|}{$\begin{array}{l}\text { Set the number of grace days where Cloudpaging player cannot } \\
\text { communicate with the server }\end{array}$} & Reduce long term Seat consumption (equivalent to license consumption) \\
\hline Concurrent per user & \multicolumn{2}{|c|}{ Set the number of executable same AppSet concurrently by one user } & $\begin{array}{l}\text { Control the number of applicatons that can be started according to the } \\
\text { software contract (one instance per one user, etc.) }\end{array}$ \\
\hline Patch Version & \multicolumn{2}{|c|}{ Set the version of updated AppSet (called "Patch Version") to be deliverd } & Force to update software \\
\hline License Type : Floating & \multicolumn{2}{|c|}{$\begin{array}{l}\text { If set, one seat is consumed where AppSet is "Starled" by Cloudpaging } \\
\text { player }\end{array}$} & Perform the operation of streaming the application from cloudpaging server \\
\hline License Type : Fixed & \multicolumn{2}{|c|}{$\begin{array}{l}\text { If set, one seat is consumed where AppSet is "Delivered" by Cloudpaging } \\
\text { server }\end{array}$} & $\begin{array}{l}\text { Perform the operation of download the application from cloudpaging server. } \\
\text { Essential parameter when Appset runs on BYOD }\end{array}$ \\
\hline $\begin{array}{l}\text { Run Option: } \\
\text { Prefetch For offline }\end{array}$ & \multicolumn{2}{|c|}{$\begin{array}{l}\text { If set, Cloudpaging player performs download all AppSet data instead of } \\
\text { streaming }\end{array}$} & $\begin{array}{l}\text { AppSet will be downloaded (it stop to streaming) from cloudpaging server. } \\
\text { Essential parameter when Appset runs on } B Y O D \text {. }\end{array}$ \\
\hline $\begin{array}{l}\text { Run Option: } \\
\text { Remove on Automatically }\end{array}$ & \multicolumn{2}{|c|}{$\begin{array}{l}\text { If set, delivered AppSet will be deleted where it exceeds "Expiration Time" } \\
\text { parameter }\end{array}$} & To simple operation, eliminates the need for user to delete AppSet \\
\hline $\begin{array}{l}\text { Principal: } \\
\text { usage right management }\end{array}$ & Behavior & \multicolumn{2}{|c|}{ Example scenario } \\
\hline User / User Group & $\begin{array}{l}\text { Use and browse permission of AppSet to particular } \\
\text { users / groups }\end{array}$ & \multicolumn{2}{|c|}{ Make AppSet available to students of a specific lecture or exercise } \\
\hline Machine / Machie Group & $\begin{array}{l}\text { Use and browse permission of AppSet to particular } \\
\text { PCS }\end{array}$ & \multicolumn{2}{|c|}{ Make AppSet available to specific classrooms } \\
\hline
\end{tabular}

Figure 4. Major parameters on meta license and principal.

2) Network connection must be campus wireless LAN.

We define the following as a Cloudpaging Meta license and Principal suitable for this usage.

\section{Applications should be prepared}

Applications matches any of the following are selected. In the practicality verification in the next section, selection of applications is made based on this criterion.

- Want to use as teaching materials, but the installation procedure is complicated and requires know-how

- Comprehensive license agreement, high usage rate as teaching materials

- Includes installation files/license information that cannot be show to students

- Requires management of usage rights (to reduce the number of simultaneous activations, uninstallation is required at graduation, etc.)

- An environment almost same as served on a PC terminal of the past educational systems

\section{Parameters for AppSet}

Parameters for AppSet (shown on Figure 4) are determined as follows.

- Prefetch for Offline: "True", License Type: "Fixed"

It is not realistic to stream AppSet to laptop under wireless LAN connection from the viewpoint of bandwidth and stability. Therefore, operated by downloading AppSet to laptop will be determined.

- Expiration Time: Set to "End of school year"

Set expiration date to prevent taking out software

- Concurrent per user: Set to "1 (For example)"

Reduce the number of simultaneous executable same AppSet per user depending on the number of software licenses

\subsection{Selection of Applications to Make AppSet}

Based on the above criterion for "Applications should be prepared", select applications and make AppSet. Since application virtualization service has just 
been launched to staffs/students of our institute, it is in the stage of practicality verification. Therefore, the selection of applications is based on the selection by administration team of educational systems. In order to improve the practicality, it is necessary to make selections based on interviews with the teacher in charge of lectures. This is major future work.

There are 30 types of applications that actually created AppSet, as shown on Figure 5. 26 types of 30 AppSet (Figure 5. Type 1,3) was created assuming general application distribution by installer operations, file layout, process access right control, etc.

For the remaining 4 types (Figure 5. Type 2) was created assuming an environment distribution for running Ubuntu Desktop on Oracle VirtualBox. Because Ubuntu Desktop was used for collective education environment on the past educational systems. Ubuntu on VirtualBox will be alternatives that.

\subsection{Practicality Verification/Judgement}

As practicality verification of application virtualization system, some measurements and judgements are made to each AppSet.

These are: 1) Measurement of delivery time (bandwidth) of AppSet to users' environment, 2) Observation of delivery successful/failed delivery AppSet on users' environment, 3) Observation of successful/failed launch of delivered AppSet as windows application on users' environment.

As users' environment, Cloudpaging Player was installed on a laptop PC (FMV-UH90, Fujitsu Limited). The network connection for users' environment was a wired LAN (1 Gbps) and wireless LAN infrastructure of our institute (IEEE802.11ac, $5 \mathrm{GHz}$ band, $300 \mathrm{Mbps}$ ). Results for verification/judgement are shown on Figure 5.

On Figure 5, time to delivery and boot up application, software profiles (name, version, purpose), size of AppSet, requirements for licensing, some important remarks except software installation process of each virtualized application are shown.

Also, Category 1 is the application that is determined to be usable, Category 2 is the distribution result of running Ubuntu Desktop on Oracle VirtualBox, and Category 3 is the application that is determined to be unusable.

\subsection{Summary of Practicality Verification}

It was determined that 25 of the 30 types could be delivered and the application could operate normally. However, even applications that are determined to be able to operate normally, some software does not operate correctly even if an AppSet is created solely by the installer. As an example, setting file allocation and access control to local programs (Figure 5, 1 - 14, Eclipse) was needed. From these results, it is a fact that the creation of AppSet is based on trial and error.

For Microsoft Office, some software and functions did not work (such as font loading and ODBC cannot be used), and problems such as destroying the Office 



Figure 5. Virtualized applications and verification results.

environment installed in the user environment were confirmed. We concluded that virtualization was difficult. For Microsoft Visual Studio, it failed in executing the command to register the product ID when creating the AppSet. We also concluded that virtualization was similarly difficult. 
With regard to the distribution of Ubuntu Desktop on Oracle VirtualBox, there are some cases where the problem is not resolved (in particular issue of USB device driver) even after trial and error (Figure 5, 2-1), or distribution is possible but it takes much distribution time (Figure 5, 2-2). Judgment of both practicality and operational policy requires careful consideration, so will be our future work.

In the wireless LAN environment, the time required from distribution to boot up application is about 2 to 3 seconds for small scale (AppSet file size is several $\mathrm{MB}$ ) software and about 3 minutes for medium scale (AppSet file size is $1 \mathrm{~GB}$ ) software.

However, it takes about 27 minutes for MATLAB (12.9 GB) with the largest AppSet file size. It is possible to use in a lecture if it is necessary to complete the software distribution before the lecture.

\section{Conclusion}

In this paper, we described an application virtualization system (Cloudpaging) adopted in an educational system to support bring and use students' laptops (BYOD) in our institute. Since practicality needs to be verified in an actual system, in this paper 30 types of applications were selected and their availability was confirmed. As a result, the delivery of the application in various installation forms and usage forms was confirmed, and the effectiveness of the application virtualization system was shown.

In the future, it will be necessary to create further operation examples, select software, and establish an operation check procedure.

\section{Conflicts of Interest}

The authors declare no conflicts of interest regarding the publication of this paper.

\section{References}

Fukuda, et al. (2019). Wireless LAN Communication Experiments for the Development of a BYOD Environment. The Internet and Operation Technologies by Constructing Resilient Information Systems. IPSJ Journal of Information Processing. (In Japanese)

Hayashi, et al. (2019). Updating the Computer System for Education and Research with a Focus on Supporting the Need for Laptops. IPSJ SIG Technical Report, 2019-IOT-46. (In Japanese)

Kai, et al. (2019). Report on the Initial Setup Workshop 2018 for Laptop Users. Bulletin of Kyushu Institute of Technology Information Science Center No. 31. (In Japanese)

Kyushu Institute of Technology (2019). Kyutech BYOD (Bring Your Own Device). (In Japanese) http://www.kyutech.ac.jp/student-cheer/kyutech byod.html

Nakamura, T. (2010) Introduction of Windows Applications Using Diskless PCs. Bulletin of Kyushu Institute of Technology Information Science Center, No. 22. (In Japanese)

Numecent Inc. (2018). Cloudpaging. https://www.numecent.com/cloudpaging/

Ohashi, et al. (2018). BYOD in Kyushu Institute of Technology. Bulletin of Kyushu Insti- 
tute of Technology Information Science Center No. 30. (In Japanese).

Tomishige, et al. (2018). An Attempt to Evaluate the Performance of the Recommended Specification of the Notebook Computer. 2018 Annual Conference of AXIES, MP-11. (In Japanese). 